\title{
Scolia
}

Revue de linguistique

\section{Les limites de la comparaison entre les parties spatiales et les parties temporelles}

Éléments de réflexion

Limits of Comparison between Spatial and Temporal Parts: Elements for Consideration

\section{Pavel Orlov}

\section{OpenEdition}

\section{Journals}

Édition électronique

URL : http://journals.openedition.org/scolia/768

DOI : $10.4000 /$ scolia.768

ISSN : 2677-4224

\section{Éditeur}

Presses universitaires de Strasbourg

\section{Édition imprimée}

Date de publication : 11 juillet 2019

Pagination : 43-59

ISBN : 979-1-03440-050-8

ISSN : 1253-9708

Référence électronique

Pavel Orlov, "Les limites de la comparaison entre les parties spatiales et les parties temporelles », Scolia [En ligne], 33 | 2019, mis en ligne le 11 juillet 2019, consulté le 11 juillet 2019. URL : http:// journals.openedition.org/scolia/768; DOI : 10.4000/scolia.768 
SCOLIA 33/ 2019, p. 43-59.

\title{
Les limites de la comparaison entre les parties spatiales et les parties temporelles Éléments de réflexion
}

\author{
Pavel OrLov \\ Université Jean Moulin Lyon 3, \\ Centre d'Études Linguistiques EA 1663 \\ pavel.orlov@univ-lyon3.fr
}

\section{Introduction}

Dans de nombreux travaux en sémantique, l'expression du temps est analysée de manière comparable à celle de l'espace. Des similitudes entre ces dimensions, au sein desquelles notre monde est inscrit, ont été repérées dans des études comparant la nature des noms et des verbes (Kleiber, 1994; Nicolas, 2002; Uspenskij, 2004). Par exemple, Krifka (1989: 76) fait la remarque suivante concernant ces catégories grammaticales:

[...] un SN comptable comme un livre dénote un objet ayant des limites précises, tout comme assembler un puzzle dénote un événement ayant des limites précises. D’autre part, un SN massif comme la bière dénote quelque chose sans une limite évidente, tout comme ce qui est dénoté par marcher n’a pas de limite évidente ${ }^{1}$.

1 «[...] a quantized NP like a book denotes an object with precise limits, just as solve the puzzle denotes an event with precise limits. On the other hand, a cumulative NP like beer denotes something without clear limitation, just like what walk denotes also has no clear limitation» (Krifka, 1989: 76), nous traduisons. 
Krifka traite donc les limites spatiales des entités concrètes un livre et la bière de la même manière que les limites temporelles des événements assembler un puzzle et marcher. Dans cet extrait, nous pouvons constater le traitement similaire accordé aux dimensions spatiales et temporelles par l'auteur.

Si l'expression du temps est comparable à l'expression de l'espace, il doit alors être possible de relever leurs similitudes au moyen de l'analyse de plusieurs types de relations sémantiques. Dans le cadre de cet article, nous souhaitons nous intéresser à l'expression de ces dimensions en nous concentrant sur la relation de partie à tout, ou méronymie, en français. Celle-ci est le reflet linguistique des relations partitives entre des référents, selon la définition de Cruse (1986: 159)2 ${ }^{2}$. Dans un nombre important de recherches, les auteurs remarquent que la méronymie peut lier des unités lexicales dénotant des entités essentiellement temporelles. Par conséquent, ils accordent une attention toute particulière aux notions d'événements et de sous-événements correspondants (Cruse, 1979, 1986, 2000; Winston et al, 1987; Vieu, 1991; Aurnague, 2004; Champollion, 2010; Champollion et Manfred, 2016; pour n'en citer que quelques-uns). Ainsi, des couples lexicaux mettant en jeu les dimensions temporelles, tels que atterrissage/ vol ou freiner / conduire, reçoivent un traitement en termes de méronymie au même titre que des couples lexicaux prototypiques roue/ voiture ou toit/ maison, opérant dans les dimensions spatiales des référents. Dans la plupart des travaux portant sur la méronymie et se concentrant sur les dimensions spatiales et temporelles, les auteurs ont donc mis l'accent sur les similitudes de ces deux dimensions.

Cependant, l'expression linguistique de la relation partie-tout entre événements, temporels par essence, n'est pas tout à fait comparable à l'expression des parties des entités spatiales. Winston et al. (1987: 426) remarquent, notamment, que la méronymie entre les événements ne peut pas être exprimée en anglais par des constructions du type «X has $Y »$. En français, cette opposition existe également. L'appartenance des parties concrètes n'est pas exprimée de la même manière que l'appartenance des parties temporelles, et ce même lorsqu'elles sont exprimées par des syntagmes nominaux:

2 «The semantic relation between a lexical item denoting a part and that denoting the corresponding whole will be termed meronymy [...]». Cruse (1986: 159). 

1) une table a des pieds
2) *un vol a un atterrissage

Aucune comparaison approfondie des relations partitives spatiales et temporelles n'a toutefois été menée à ce jour. C'est sur cette analyse que porte le présent article. Notre comparaison des méronymies spatiales et temporelles s'articule selon trois principaux axes. Tout d'abord, dans la section 1, nous explorerons la possibilité pour le locuteur d'accéder aux dimensions temporelles des entités spatiales et vice versa. Nous développerons ensuite, dans la section 2, l'orientation pouvant être attribuée aux entités-touts aussi bien spatiales que temporelles. Pour finir, dans la section 3, nous nous concentrerons sur les manières de localiser les parties spatiales et temporelles au sein des entités-touts correspondantes. Nous arriverons à la conclusion que, pour chacun de ces trois aspects, il existe des asymétries conceptuelles entre l'espace et le temps pour un locuteur, ce qui a été ignoré dans la littérature existante.

\section{Accès aux dimensions sous-jacentes}

Dans la présente section, nous explorerons la possibilité pour un locuteur d'accéder aux dimensions temporelles des entités essentiellement spatiales et aux dimensions spatiales des événements.

\subsection{Parties temporelles des entités concrètes}

Même si les objets concrets sont a priori ancrés dans le domaine spatial, ils ne sont pas pour autant dénués de dimension temporelle. Les entités matérielles sont indirectement associées à des événementsétats. Ainsi, Aurnague (1998: 22) parle de «la durée de vie» d'un objet, c'est-à-dire de la période temporelle correspondant à son existence. Hayes (1978, 1985), dont le travail a été cité par Vieu (1991: 78) parle d' "histoire» afin de traduire le même concept. Ces notions sont largement utilisées en logique des relations temporelles.

Toutefois, dans la majorité des cas, la langue n'autorise pas le locuteur à accéder au versant temporel des objets concrets $\left({ }^{*}\right.$ durant Pierre, ${ }^{\star}$ lors de la tasse), comme l'a remarqué Huyghe (2012). Nous pouvons ajouter à cette remarque qu'il est également impossible de créer des parties temporelles des entités spatiales. Ainsi, il n'est pas 
acceptable de désigner une période arbitraire dans les limites de la durée de vie d'une entité ( ${ }^{*}$ cinq minutes de la table, ${ }^{\star}$ deux jours $d u$ mur) ni même leurs différentes "phases» ( ${ }^{*} l e$ début $d u$ sac, ${ }^{\star} l a$ fin $d u$ vase).

Les noms des êtres animés, et notamment les noms d'humains, constituent sans doute un cas à part car ils permettent, a priori, d'accéder aussi bien au domaine spatial que temporel de leurs référents. Effectivement, la notion de «durée de vie» que nous venons d'exposer est plus saillante pour les animés. Leur spécificité permet la référence à une multitude de périodes associées (Alexandrova, 2013):

3) l'enfance / l'adolescence / la vieillesse de Pierre

Les noms de ces périodes permettent au locuteur d'accéder à la dimension temporelle des êtres animés. Toutefois, malgré l'existence de ces unités lexicales, les périodes de vie arbitraires, désignées à l'aide de noms d'unités temporelles (heure, jour, année, etc.), ne peuvent en principe être exprimées:

4) ${ }^{*}$ cinq minutes $/{ }^{*}$ dix heures de Pierre ${ }^{3}$

Cela signifie que le locuteur peut accéder à certaines périodes associées aux êtres animés, lorsqu'elles sont lexicalisées, sans pour autant pouvoir se référer à leur dimension temporelle sous tous ses aspects. Les parties désignées par des noms d'unités temporelles peuvent être employées, lorsque le locuteur précise de manière explicite qu'il s'agit de la dimension temporelle de l'animé en question:

5) cinq minutes / dix heures de la vie de Pierre

En effet, dans la construction ci-dessus, l'entité-tout est désignée par la vie de Pierre et non par Pierre. Il s'agit donc d'un événement associé à Pierre, ce qui permet l'interprétation partitive temporelle.

Il semble donc que de manière générale, il est impossible pour un locuteur de se référer à des parties temporelles des entités spatiales.

3 Ce type de constructions peut être trouvé avec les compléments locatifs: deux ans de Pierre dans le cabinet notarial (ont suffi à bouleverser l'organisation). Toutefois, l'analyse de ce type d'énoncés nous éloigne de la problématique des relations partitives. 


\subsection{Parties spatiales des entités temporelles}

Les événements, quant à eux, se situent principalement dans le temps, mais peuvent avoir une dimension spatiale. En effet, la participation d'agents matériels à un événement permet souvent de le situer dans le domaine spatial. Ainsi, dans une maison brûle, la girafe boit ou un trésor gît l'action ou l'état désigné a lieu quelque part. De même, les noms d'événements peuvent faire partie des constructions locatives, comme dans la conférence a lieu à Toulouse ou la cérémonie se déroule à la Sorbonne. Sont évidemment exclus de ce cas de figure les événements qui, bien qu'ayant des sujets concrets, présentent une localisation inconnue ou non pertinente, comme dans Pierre aime les fruits, Marie a perdu sa montre, etc.

Mais s'il est possible de localiser des événements dans l'espace, ils n'ont généralement pas d'extension spatiale, comme le soutient Huyghe (2012: 7). Ainsi des expressions comme *la superficie d'un mariage, *le périmètre d'une réunion ou *la largeur d'un match ne sont pas acceptables, contrairement à la superficie d'un champ, le périmètre d'une roue ou la largeur d'une table. En l'absence d'extension physique des événements accessible pour le locuteur, il lui est, plus généralement, impossible de procéder à un quelconque découpage spatial de ce type d'entités:

6) *le nord de l'inauguration

7) *le centre d'une conférence

8) *un mètre carré d'un concert

Il existe cependant des exceptions, et certains noms d'événements peuvent faire référence à des portions de l'espace correspondant à leur extension spatiale. Il s'agit vraisemblablement d'événements auxquels un espace, présentant parfois une délimitation précise, peut être attribué, comme dans le cas des noms festival ou exposition. On trouve également dans cette catégorie des noms tels que course ou randonnée, pouvant faire référence aussi bien à l'activité elle-même qu'au parcours, ou tracé, de cette dernière. Or, étant donné l'attribution d'un espace à des événements de ce type, des parties spatiales peuvent être distinguées comme dans les exemples ci-dessous:

9) le moindre mètre carré du festival est exploité (<www. radiometal.com>, 11/04/2018) 
10) j'ai mis mes qualités à contribution dès les premiers mètres de la course (<www.lequipe.fr>, 11/04/2018)

11) l'enneigement est plus important dans la partie sud de la randonnée (<www.partance.org>, 11/04/2018)

Dans certains contextes, il est même possible de rencontrer un découpage en parties spatiales d'événements qui ne présupposent aucune extension fixe, ou préalablement établie, de ce type. À titre d'exemple, un locuteur commente un match de rugby de la manière suivante:

12) il manquait un peu de gaz sur les derniers mètres de son essai (<www.forum.fcgrenoble.com>, 11/04/2018)

Le locuteur utilise ici le nom essai pour désigner le parcours du sportif dans le cadre de l'événement «marquer l'essai», sans que ce parcours ait été physiquement établi au préalable. Le découpage spatial de cet événement est acceptable, bien que son extension physique ne soit pas aussi immédiate que dans les cas d'une randonnée ou d'une course: à comparer la longueur de la randonnée et ??la longueur de l'essai.

En résumé, contrairement aux noms d'entités spatiales, les noms d'événements permettent au locuteur de se référer à leurs domaines spatial et temporel. Il s'agit ici d'une première asymétrie entre les parties spatiales et temporelles, que nous dégageons dans ce travail.

\section{L'orientation des entités-touts}

\subsection{Définition de l'orientation}

Certaines entités peuvent être perçues comme intrinsèquement orientées. Vandeloise (1986a, 1986b) parle de l'orientation générale des entités définie en fonction de leur structure interne (les symétries et les asymétries du corps), du positionnement des organes de perception (en particulier des organes de vision) et/ou de la direction de leurs déplacements prototypiques ${ }^{4}$. Ainsi, les organes de sens orientent la plupart des êtres animés: c'est la raison pour laquelle les expressions devant un chien / un oiseau désignent en principe toujours le même

4 Nous pouvons ajouter à ces critères la position de la personne qui les utilise (un écran, un canapé) ou la position prototypique de cette entité (une montagne), qui sont, d'une certaine façon, des cas particuliers de l'orientation générale. 
espace par rapport à l'animal, indépendamment de la position du locuteur: il s'agit d'une portion de l'espace devant sa gueule ou son bec. Le sens du déplacement, quant à lui, définit l'orientation de véhicules. Bien qu'une voiture puisse faire marche arrière, l'avant et l'arrière de ce véhicule sont définis par le sens de son déplacement prototypique (Vieu, 1991: 17).

\subsection{Orientation des parties spatiales}

La dénomination de certaines parties tient compte de l'orientation générale de l'entité-tout (pour l'axe frontal) et, plus généralement, de son/ses orientation(s) intrinsèque(s). Ceci est vrai pour les Noms de Localisation Interne orientationnels ${ }^{5}$ (par exemple avant, arrière, haut, bas, etc.) qui relèvent, selon Aurnague (1996: 2), de la relation de méronymie. On parle ainsi de l'avant d'une voiture, du bas d'un escalier ou du haut d'une armoire. Outre ces NLI génériques, il existe des noms de parties orientées spécifiques à certaines entités: par exemple, les référents de poupe et proue sont définis en fonction de l'orientation générale d'un bateau. Les noms d'entités dépourvues d'orientation(s) intrinsèque(s) ne sont pas compatibles avec les NLI ou nécessitent une interprétation de ces lexèmes dans laquelle l'orientation est attribuée contextuellement: *l'avant / *l'arrière d'un grain de riz / d'une soupe, *le haut / *le bas d'un mètre ruban / d'une pelouse, etc.

Le NLI bout est un bon indicateur lexical de l'orientation des entités unidimensionnelles ou pouvant être assimilées à de telles entités. Tout d'abord, dans le cas des entités non-orientées de manière intrinsèque - comme dans le bout d'une corde / d'un bâton - il désigne, généralement, l'une des deux extrémités longitudinale de l'objet en question. Il est d'ailleurs possible de décrire ces entités comme suit:

13) Une corde / un bâton a deux bouts

À l'inverse, dans le cas des entités intrinsèquement orientées, le mot bout désigne une seule extrémité. Par exemple, il peut dénoter l'extrémité fonctionnelle dans le bout d'un couteau ou la partie la plus avancée dans l'eau dans le bout d'une jetée. Tout comme un bâton, un couteau possède deux extrémités d'un point de vue topologique: une

5 Nous ne couvrirons que ce type de Noms de Localisation Interne dans cet article, et les désignerons ci-dessous par «NLI», selon les conventions habituelles. 
du côté de la lame et l'autre du côté du manche. Cependant, l'usage que l'on fait d'un couteau lui donne une orientation.

De toute évidence, certaines situations d'énonciation peuvent attribuer une orientation pragmatique à des entités intrinsèquement non-orientées, comme bâton, vu précédemment. Le nom bout peut alors tout à fait être employé avec un article défini et désigner une extrémité bien précise de l'entité en question:

14) [...] avec le bout d'un bâton on titille un poisson échoué pour s'assurer qu'il est bien mort. (Vilaine, Le silence ne sera qu'un souvenir, 2011)

Le bâton n'est pas perçu dans ce cas comme un simple objet, mais comme un outil auquel on affecte une fonction. Ici, plus spécifiquement, le bâton de (14) sert à toucher ou déplacer le poisson sans que l'on ait à le manipuler; la partie du bâton dénommée par bout est alors la partie fonctionnelle, c'est-à-dire celle qui entre en contact avec le poisson.

Un mécanisme pragmatique similaire permet de faire la différence entre les énoncés suivants:

15) j'aperçois l'entrée du tunnel

16) j'aperçois le bout du tunnel

Dans ces deux exemples, le locuteur peut désigner une même partie du tunnel par les noms entrée et bout. Cependant, ils ne seront certainement pas employés dans des contextes d'énonciation similaires. En effet, on parle de l'entrée du tunnel lorsque le locuteur se trouve à l'extérieur de celui-ci et, éventuellement, lorsqu'il se dirige vers lui. Le bout du tunnel est employé plutôt pour désigner la sortie, lorsque le locuteur est à l'intérieur du tunnel.

Il apparaît à l'issue de cette section que les entités matérielles peuvent donc être orientées par essence (l'avant d'une voiture), ne pas être orientées (l'arrière d'une soupe) ou recevoir une orientation via des mécanismes pragmatiques (le bout d'un bâton).

\subsection{Orientation des parties spatiales}

Abordons à présent la possibilité pour un locuteur de percevoir l'orientation des événements. La nature du temps, dans lequel les événements sont inscrits, impose de fait un sens à ce type d'entités. Ce 
raisonnement, fondé sur les informations extra-linguistiques, trouve un écho en linguistique. En effet, la dénomination des extrémités temporelles des événements non-ponctuels tient compte de cette orientation. Dans la majorité des cas, elle se fait de manière générique à l'aide des noms début et fin:

17) le début d'une intervention / bagarre / réparation

18) la fin d'un incendie / repas / cours

Pour un certain nombre de noms d'événements, la dénomination de ces extrémités temporelles peut être plus spécifique. On emploie, par exemple, les noms décollage et atterrissage pour désigner le début et la fin de l'événement vol, ou encore les noms naissance et mort pour ceux de l'événement vie, etc. Il est intéressant de noter que atterrissage n'est méronyme que de $\mathrm{vol}$, car il n'existe pas d'autres événements-touts au même niveau hiérarchique qu'un vol dont un atterrissage pourrait faire partie. De même, une naissance est toujours un moment, ou une phase, d'une vie. On peut dire que ces noms de parties n'ont pas d'entitétout alternative ${ }^{6}$ ce qui rend douteuse l'acceptabilité des constructions suivantes:

19) ??l'atterrissage d'un vol

20) ??la naissance d'une vie

Pour que ces constructions soient acceptables, il est nécessaire que des entités-touts alternatives existent. Cette condition est remplie, par exemple, lorsque les syntagmes génériques en (19) et (20) sont remplacés par des constructions ayant une lecture spécifique:

21) l'atterrissage du vol AF3321 / d'un Airbus A330

22) la naissance de Pierre / du chat

Il existe en effet, au même niveau que le vol AF3321, d'autres entités-touts dont l'atterrissage peut faire partie. Remarquons que, dans le cas de (22), la référence à un événement vie particulier passe par la référence à l'être animé correspondant.

Le NLI bout, dont l'usage est différent avec des entités-touts spatiales orientées et non-orientées, peut également se rapporter à des

6 Cruse (2000: 152) utilise la notion de congruence de portée (range congruence) pour décrire ce type de couples lexicaux partie/tout. Raxilina (2008:42) parle de fixité dénotationnelle d'une relation partie-tout (denotativno fiksirovannoe otnošenie). 
parties temporelles d'événements. Il désigne, dans la majorité des cas, la fin d'un événement, notamment lorsqu'il est associé aux verbes arriver et parvenir dans des constructions arriver / parvenir au bout de ${ }^{7}$ :

23) arriver au bout de la compétition / d'un morceau (musical)

24) parvenir au bout de la mission / formation

Dans tous ces cas, les événements dénotés n'ont pas de domaine spatial bien défini et seule la lecture partitive temporelle est possible: bout désigne un stade du procès - la fin de l'événement-tout - et non un espace quel qu'il soit. Ce type de constructions reste bien sûr acceptable avec les noms d'événements présentant un versant spatial, en sus de leur dimension temporelle:

25) Gagner une course, c'est arriver au bout. (<https://www. ladepeche.fr $>, 11 / 03 / 2018$ )

L'existence d'un tracé de la course - c'est-à-dire d'un espace qui lui est réservé - implique la possibilité d'une lecture partitive spatiale de la relation entre bout et course. Cet énoncé présente une anaphore associative méronymique, pour reprendre les termes de Kleiber (1999). En effet, le couple lexical bout / course est lié par une relation de méronymie, étant donné le statut de NLI de bout. Arriver au bout (de la course) véhicule le sens "franchir la ligne d'arrivée» et non «se positionner au point de départ». Le NLI bout appliqué aux événements de ce type a également un seul référent spatial - le site localisé à la fin du déplacement - dans des constructions sans les verbes arriver/venir:

26) le bout de la course / randonnée (est encore loin)

Dans certains cas, le mot bout renvoie à n'importe quelle extrémité existante:

27) les équipes médicales se trouvent à chaque bout de la course

28) à chaque bout de la randonnée se trouve un parking

Cette utilisation de bout indique alors quel'entité-tout est perçue comme non-orientée. Cela ne remet pas en cause l'orientation intrinsèque des événements déjà mise en évidence, car dans ces cas l'entité-tout relève uniquement du versant spatial de course et randonnée. Dans (28) par exemple, randonnée désigne non pas une activité ou un événement,

7 Le même phénomène peut être observé avec les constructions venir à bout de. Nous ne la traitons pas ici à cause de son caractère figé. 
mais un circuit pouvant être emprunté par des personnes. Toute interprétation événementielle de ce nom est d'ailleurs exclue en présence de la construction à chaque bout de, ce qui rend difficilement acceptables les exemples ci-dessous:

29) ??l'athlète a bu de l'eau à chaque bout de la course

30) ??nous avons pris des photos à chaque bout de la randonnée

Tous ces exemples nous portent à croire que, contrairement aux entités matérielles, les événements sont toujours conceptualisés comme orientés, et exprimés tels quels, par les locuteurs. Dès lors qu'un nom d'événement est présenté sous une perspective non-orientée, et notamment lorsque la distinction début / fin n'est pas faite, la référence qui s'opère a trait à son domaine spatial uniquement. Il s'agit d'une deuxième asymétrie entre les parties spatiales et temporelles que nous relevons dans cet article.

Une attention particulière doit être accordée à la construction d'un bout à l'autre, comme dans les exemples suivants:

31) l'athlète doit rester dans le couloir d'un bout à l'autre de la course

32) nous avons marché seuls d'un bout à l'autre de la randonnée

Remarquons que les entités-touts course et randonnée semblent ici présentées comme non-orientées, aucune distinction lexicale n'étant explicitement faite entre leurs débuts et leurs fins ${ }^{8}$. Toutefois, l'interprétation en jeu est strictement événementielle et non spatiale. Ces énoncés sont alors à distinguer des exemples suivants, ayant une lecture spatiale:

33) L'appel des guetteurs résonna d'un bout à l'autre des remparts. (Moore, Le peuple du vent: Les aventures de Tancrède le Normand, 2011)

34) une émission de télévision diffusée d'un bout à l'autre du pays. (MacDonald, Un trombone rouge, 2011)

C'est en fait à deux emplois distincts de d'un bout à l'autre que l'on est ici confrontés. La lecture temporelle de cette construction peut d'ailleurs être facilement mise en évidence en la remplaçant par des prépositions véhiculant l'idée de durée:

8 La différentiation lexicale début/fin est toutefois implicite, car la première occurrence de bout désigne le début, tandis que la seconde - la fin. 
35) l'athlète doit rester dans le couloir durant la course

36) nous avons marché seuls pendant la randonnée

37) *l'appel des guetteurs résonna durant les remparts

38) *une émission de télévision diffusée pendant le pays

Le cas de d'un bout à l'autre constitue, à notre connaissance, la seule exception permettant une lecture non-orientée d'une entité temporelle.

\section{La localisation des parties}

Les relations partitives impliquant des NLI orientationnels, abordés dans la section précédente, désignent des parties dont la position au sein des entités-touts est bien définie. Par exemple, le nom bout est lexicalement défini comme "portion extrême d'une chose considérée comme un continu allongé» (TLFi, 12/04/2018). Or, ainsi que le remarque Borillo (1988) ${ }^{9}$, «dans le cas le plus courant, un nom désignant une partie d'objet, n'indique rien sur la position que cette partie occupe par rapport aux autres ». C'est pourquoi, dans le discours, il est permis à un locuteur de préciser la localisation d'une partie par rapport à son tout.

\subsection{Localisation des parties spatiales}

Dans le cas des entités matérielles, la localisation se fait généralement à l'aide de prépositions locatives telles que dans ou sur:

39) Tu vois le nombre de feuilles sur cet arbre? (Jollien, Le Philosophe nu, 2010)

40) Le tiroir dans le meuble est refermé d'un coup sec. (Monnier, Le presbytère, 2017)

Une ambiguité existe entre l'interprétation partitive ou locative de ce type d'énoncés. Mikaelian (2002: 198) avance la thèse selon laquelle «les prépositions spatiales n'expriment pas directement la relation "partie-tout": leur fonction consiste à former des "places" et à préciser la configuration spatiale des objets autonomes les uns par rapport aux autres». Néanmoins, Winston et al. (1987) ainsi que Kleiber (1997: 47) ont défendu l'idée selon laquelle il existe, aussi bien en français qu'en

9 Cité dans Aurnague (2004: 95). 
anglais, une distinction entre le phénomène de localisation des parties par rapport à leurs touts et la relation de localisation spatiale des entités indépendantes. Les exemples (39) et (40) ne sont alors pas à confondre, d'un point de vue sémantique, avec les énoncés suivants:

41) le livre (est) sur la table

42) la voiture (est) dans le garage

En effet, dans l'exemple (39) feuille est interprété comme une partie de l'entité-tout arbre, alors que dans l'exemple (41) livre est une entité indépendante de table.

\subsection{Localisation des parties temporelles}

À l'instar de la localisation des entités matérielles dans l'espace, la localisation des entités temporelles sur l'axe du temps est également possible. Les événements peuvent ainsi être situés dans le temps par rapport à d'autres événements, notamment via l'indication de la simultanéité (Aslanides Rousselet, 1998) : elle téléphonait en conduisant, il réfléchissait pendant qu'il marchait. Cependant, l'expression de la simultanéité entre les événements-touts et leurs sous-événements semble être un mécanisme peu usité par les locuteurs. Premièrement, les noms de sous-événements véhiculent, dans un certain nombre de cas, des informations sur la localisation temporelle de ces derniers au sein de l'événement-tout correspondant. Ceci est notamment vrai pour les noms des segments initiaux ou finaux de certains événements tels que naissance ou atterrissage étudiés précédemment. Leur localisation temporelle au sein de leurs événements-touts paraît alors tautologique:

43) *l'enfance lors / au cours de la vie

44) *l'atterrissage à la fin $d u$ vol

En effet, il est tautologique de situer l'enfance dans les limites temporelles d'une vie, car l'existence de l'entité temporelle enfance présuppose l'existence de l'entité-tout vie. De même, tout atterrissage fait nécessairement partie d'un vol.

Deuxièmement, d'autres événements-touts sont composés d'événements cycliques élémentaires: les référents de marcher et courir consistent en une série de pas, nager désigne également une action composée (une série de brasses). Dans ces cas de relation partie-tout, il existe des mouvements élémentaires à tout instant de l'événement- 
tout: il n'y a plus d'action de marcher dès lors qu'aucun pas n'est fait. Les énoncés ci-dessous sont donc eux aussi tautologiques et ne sont pas attestés en français:

45) *il faisait des pas en marchant

46) *il faisait des pas au début / à la fin de la marche

47) *en marchant il faisait des pas sans arrêt / tout le temps

Troisièmement, certains sous-événements, tels que freinage pour conduite ou mancuvre pour vol, n'ont pas de position temporelle bien définie au sein de l'événement-tout car ils peuvent se produire à tout moment du déroulement de ce dernier. Ces parties temporelles semblent même facultatives: il est possible que des événements de conduite spécifiques ne comportent pas de freinage, et des occurrences de vols peuvent être également dénuées de toute manœuvre. Ce type de parties n'est généralement pas présenté comme simultané avec le tout (48), contrairement à des événements indépendants (49):

48) ??elle a dû freiner pendant qu'elle conduisait

49) Elle a dî prendre une décision pendant qu'elle conduisait.

(Chesbro, Hémorragie dans l'œil du cyclone mental, 2013)

Il est toutefois possible de préciser la localisation temporelle du sous-événement au sein de l'événement tout, comme dans (50), ou bien de préciser la fréquence de ses multiples occurrences, comme dans (51):

50) elle a fait une manœuvre au début du vol

51) en conduisant elle freinait sans arrêt / tout le temps

Comme nous pouvons le constater à partir des exemples précédents, les parties des entités matérielles semblent pouvoir être localisées dans l'espace occupé par leurs touts. A contrario, les sous-événements peuvent être localisés dans les domaines temporels des événementstouts seulement de manière occasionnelle.

\section{Conclusion}

Dans son travail, Huyghe (2012) a mis en évidence des asymétries dans la représentation linguistique de l'espace et du temps. Cela va à l'encontre du postulat, selon lequel l'expression du temps doit être analysée de manière strictement comparable à celle de l'espace. 
Dans cet article nous nous sommes intéressé à l'expression de ces deux dimensions dans le cadre bien précis de la méronymie en français. Les précédents travaux portant sur la méronymie (Cruse, 1979, 1986, 2000; Winston et al., 1987; Vieu, 1991; Aurnague, 2004; Champollion, 2010; Champollion et Manfred, 2016) ont mis l'accent sur les similarités entre ces deux domaines du monde référentiel. À travers l'exploration de l'accès aux dimensions sous-jacentes des entités par les locuteurs, de l'orientation et de la localisation des entités spatiotemporelles, nous avons mis en évidence des asymétries existant entre la méronymie spatiale et temporelle.

Tout d'abord, en règle générale, le locuteur ne peut désigner que des parties spatiales des entités matérielles, tandis que les noms d'événements semblent pouvoir être associés aussi bien à des parties spatiales que temporelles. Par ailleurs, les événements sont généralement perçus et décrits par les locuteurs comme des entités orientées ce qui permet une distinction entre les sous-événements initiaux et finaux. A contrario, la plupart des entités matérielles ne sont pas nécessairement orientées. Enfin, les parties des entités concrètes peuvent être localisées au sein de leurs touts, et donc occuper une même portion de l'espace qu'eux, alors que l'indication de la simultanéité d'un événement et d'un sous-événement ne semble pas être un procédé linguistique généralisé.

\section{Bibliographie}

ALEXANDROVA A. (2013), Noms humains de phase: problèmes de classifications ontologiques et linguistiques, thèse de doctorat, Université de Strasbourg.

ASLANIDES ROUSSELET S. (1998), Exprimer linguistiquement une relation entre deux événements: les connecteurs de la simultanéité en français, in Borillo A., Vetters C. \& Vuillaume M. (éds), Variations sur la référence verbale, Amsterdam, Rodopi, 277-292.

AURNAGUE M. (1996), Les Noms de Localisation Interne: tentative de caractérisation sémantique à partir de données du basque et du français, Cahiers de lexicologie 69, 159-192.

AURNAGUE M. (1998), Basque genitives and part-whole relations: typical configurations and dependences, Equipe de Recherche en Syntaxe et Sémantique (ERSS), <http://artxiker.ccsd.cnrs.fr/ artxibo-00000033>. 
AURNAGUE M. (2004), Les structures de l'espace linguistique. Regards croisés sur quelques constructions spatiales $d u$ basque et $d u$ français, Paris, Peeters.

BORILLO A. (1988), Le lexique de l'espace: les noms et les adjectifs de localisation interne, Cahiers de grammaire 13, 3-22.

CHAMPOLLION L. (2010), Parts of a Whole: Distributivity as a Bridge Between Aspect and Measurement, PhD Dissertation, University of Pennsylania.

CHAMPOLLION L. \& MANFRED K. (2016), Mereology, in Dekker P. \& Aloni M. (eds.), The Cambridge Handbook of Formal Semantics, Cambridge, Cambridge University Press, 513-541.

CRUSE D.A. (1979), On the transitivity of the part-whole relation, Journal of Linguistics $15:$ 1, 29-38.

CRUSE D.A. (1986), Lexical semantics, Cambridge, Cambridge University Press.

CRUSE D.A. (2000), Meaning in language: an introduction to semantics and pragmatics, Oxford, Oxford University Press.

HAYES P. (1978), The naive physics manifesto, in Michie D. (ed.), Expert systems in the micro-electronic age, Edinburgh, Edinburgh University Press.

HAYES P. (1985), The second naive physics manifesto, in Hobbs J. \& Moore R. (eds.), Formal theories of the commonsense world, Norwood, Ablex, 1-36.

HUYGHE R. (2012), Noms d'objets et noms d'événements: quelles frontières linguistiques?, Scolia 26, 81-103.

KLEIBER G. (1994), Nominales: essais de sémantique référentielle, Paris, A. Colin.

KLEIBER G. (1999), Anaphore associative et relation partie-tout: condition d'aliénation et principe de congruence ontologique, Langue française 122, 70-100.

KRIFKA M. (1989), Nominal Reference, Temporal Constitution and Quantification in Event Semantics, in Bartsch R., Semantics and Contextual Expression, Dordrecht, Foris, 75-111.

MIKAELIAN I. (2002), La possession en russe moderne: éléments pour la construction d'une catégorie sémantico-syntaxique, Villeneuve-d'Ascq, Atelier national de reproduction des thèses.

NICOLAS D. (2002), La distinction entre noms massifs et noms comptables: aspects linguistiques et conceptuels, Leuven, Peeters.

USPENSKIJ B. (2004), Čast' $i$ celoe v russkoj grammatike, Moscou, Jazyki slavjanskoj kul'tury. 
VANDELOISE C. (1986a), L'avant/l'arrière et le devant/le derrière, Revue québécoise de linguistique 16: 1, 281-307.

VANDELOISE C. (1986b), L'espace en français: sémantique des prépositions spatiales, Paris, Seuil.

VIEU L. (1991), Sémantique des relations spatiales et inférences spatiotemporelles: une contribution à l'étude des structures formelles de l'espace en Langage Naturel, thèse de doctorat, Université Paul Sabatier.

WINSTON M.E., CHAFFIN R. \& HERRMANN D. (1987), A Taxonomy of Part-Whole Relations, Cognitive Science 11, 417-444. 